\title{
Enclosing Ellipses by Folding Disks
}

\author{
Peter Paul Klein \\ University of Technology Clausthal, Clausthal-Zellerfeld, Germany \\ Email: peter.p.klein@t-online.de
}

How to cite this paper: Klein, P.P. (2022) Enclosing Ellipses by Folding Disks. Applied Mathematics, 13, 147-162. https://doi.org/10.4236/am.2022.132012

Received: December 28, 2021

Accepted: February 18, 2022

Published: February 21, 2022

Copyright $\odot 2022$ by author(s) and Scientific Research Publishing Inc. This work is licensed under the Creative Commons Attribution International License (CC BY 4.0).

http://creativecommons.org/licenses/by/4.0/ (c) (i)

Open Access

\begin{abstract}
Ellipses can be constructed by folding disks. These folds are forming an envelope of tangents to the ellipse. In the paper of Gorkin and Shaffer, it was shown that the ellipse constructed by folding can be circumscribed by an arbitrary triangle of tangents, the vertices of which are lying on the circumference of the disk. They offered two non-elementary methods of proof, one using Poncelet's Theorem, the other employing Blaschke products. In this paper, it is the intention to present an elementary proof by means of analytic geometry.
\end{abstract}

\section{Keywords}

Straight Line, Perpendicular Bisector, Linear System, Determinant, Point of Intersection, Gardner Ellipse, Bidirectional Folding

\section{Introduction}

The disk with which the folding is performed shall be represented by a circle $x^{2}+y^{2}=r^{2}$ with radius $r>0$ and center $C=(0,0)$. The point $D$ inside the circle and different from the center $C$ shall be described as $D=(d, 0)$ with $|d|<r$. The fold performed by laying a point $A_{0}$ of the circumference of the disk on top of $D$ can be described by the perpendicular bisector of the line segment $\overline{A_{0} D}$, where $A_{0}=\left(\xi_{0}, \eta_{0}\right)$ fulfills $\xi_{0}^{2}+\eta_{0}^{2}=r^{2}$. The straight line running through $D=(d, 0)$ and $A_{0}$ has the form

$$
\eta_{0} x-\left(\xi_{0}-d\right) y=\eta_{0} d .
$$

The perpendicular bisector of line segment $\overline{A_{0} D}$ is given by

$$
\left(\xi_{0}-d\right) x+\eta_{0} y=\frac{1}{2}\left(r^{2}-d^{2}\right) .
$$

The point of intersection of both straight lines (1) and (2) is $M_{0}=\left(\frac{1}{2}\left(\xi_{0}+d\right), \frac{1}{2} \eta_{0}\right)$. They are perpendicular to each other because their normal vectors are orthogonal: $\eta_{0}\left(\xi_{0}-d\right)-\left(\xi_{0}-d\right) \eta_{0}=0$. See Figure 1 . 


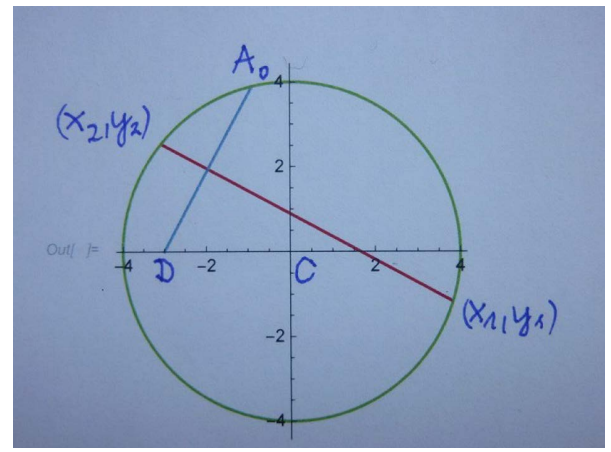

Figure 1. Perpendicular bisector of $\overline{A_{0} D}$.

The term "sequential folding" was established by Gorkin and Shaffer [1] and means: After the first fold, proceed in such a way as to start the next fold where the previous fold ended. In contrast to Gorkin and Shaffer [1] in this paper after the first fold, the second fold and the third fold are starting at the two different ends of the first fold on the circle $x^{2}+y^{2}=r^{2}$, at $\left(x_{1}, y_{1}\right)$ and $\left(x_{2}, y_{2}\right)$ respectively. This shall be named "bidirectional folding". In the second fold, $A_{1}=\left(\xi_{1}, \eta_{1}\right)$ has to be chosen on the circle $x^{2}+y^{2}=r^{2}$ in such a way that $\left(x_{1}, y_{1}\right)$ is a point on the perpendicular bisector of $\overline{A_{1} D}$ :

$$
\left(\xi_{1}-d\right) x_{1}+\eta_{1} y_{1}=\frac{1}{2}\left(r^{2}-d^{2}\right), \xi_{1}^{2}+\eta_{1}^{2}=r^{2} .
$$

In the third fold, a point $A_{2}=\left(\xi_{2}, \eta_{2}\right)$ has to be chosen on the circle $x^{2}+y^{2}=r^{2}$ such that $\left(x_{2}, y_{2}\right)$ is a point on the perpendicular bisector of $\overline{A_{2} D}$ :

$$
\left(\xi_{2}-d\right) x_{2}+\eta_{2} y_{2}=\frac{1}{2}\left(r^{2}-d^{2}\right), \xi_{2}^{2}+\eta_{2}^{2}=r^{2} .
$$

The final problem will be, to show that perpendicular bisectors of $\overline{A_{1} D}$ and $\overline{A_{2} D}$

$$
\begin{aligned}
& \left(\xi_{1}-d\right) x+\eta_{1} y=\frac{1}{2}\left(r^{2}-d^{2}\right) \\
& \left(\xi_{2}-d\right) x+\eta_{2} y=\frac{1}{2}\left(r^{2}-d^{2}\right)
\end{aligned}
$$

have a point of intersection $\left(x_{T}, y_{T}\right)$ on the circle $x^{2}+y^{2}=r^{2}$. Because of the quadratic Equations in (3) and (4) involved in the calculation of $\left(\xi_{1}, \eta_{1}\right)$ and $\left(\xi_{2}, \eta_{2}\right)$ we get two solutions $\left(\left(\xi_{1}\right)_{ \pm},\left(\eta_{1}\right)_{\mp}\right)$ and $\left(\left(\xi_{2}\right)_{ \pm},\left(\eta_{2}\right)_{\mp}\right)$ respectively. The number of solutions can be reduced, however, looking at two straight lines: one passing through $\left(\left(\xi_{1}\right)_{+},\left(\eta_{1}\right)_{-}\right)$and $\left(\left(\xi_{1}\right)_{-},\left(\eta_{1}\right)_{+}\right)$the other through $\left(\left(\xi_{2}\right)_{+},\left(\eta_{2}\right)_{-}\right)$and $\left(\left(\xi_{2}\right)_{-},\left(\eta_{2}\right)_{+}\right)$. It will be shown that the point of intersection $\left(x_{S}, y_{S}\right)$ of both straight lines is located on the circle $x^{2}+y^{2}=r^{2}$ and is equal to $A_{0}=\left(\xi_{0}, \eta_{0}\right)$.

The procedure described above constructs an arbitrary triangle circumscribing an ellipse and having vertices on a circle. This is in contrast to the approach in [1], where a special triangle was chosen to circumscribe an ellipse and have 
vertices on a circle. The generalization to an arbitrary triangle is accomplished by employing Poncelet's Theorem [2]. In a remark in [1], a different way of proof is indicated with a Blaschke product of degree three, treated more extensively in [3].

\section{Preliminaries}

Next we want to find the points of intersection of (2) with the circle $x^{2}+y^{2}=r^{2}$. Assuming $\eta_{0} \neq 0$ Equation (2) can be rewritten as

$$
y=-\frac{\xi_{0}-d}{\eta_{0}} x+\frac{r^{2}-d^{2}}{2 \eta_{0}}
$$

which gives after substitution into the equation of the circle and multiplication with $\eta_{0}^{2}$

$$
\eta_{0}^{2} x^{2}+\left(-\left(\xi_{0}-d\right) x+\frac{1}{2}\left(r^{2}-d^{2}\right)\right)^{2}-\eta_{0}^{2} r^{2}=0
$$

So we get

$$
\left(\left(\xi_{0}-d\right)^{2}+\eta_{0}^{2}\right) x^{2}-\left(\xi_{0}-d\right)\left(r^{2}-d^{2}\right) x+\frac{1}{4}\left(r^{2}-d^{2}\right)^{2}-\eta_{0}^{2} r^{2}=0 .
$$

The highest coefficient in (7) is different from zero for $\left(\xi_{0}, \eta_{0}\right)$ with $\xi_{0}^{2}+\eta_{0}^{2}=r^{2}$ because

$$
\left(\xi_{0}-d\right)^{2}+\eta_{0}^{2}=r^{2}-2 \xi_{0} d+d^{2} \geq r^{2}-2 r|d|+d^{2}=(r-|d|)^{2}>0 .
$$

So we get

$$
x^{2}-\frac{\left(\xi_{0}-d\right)\left(r^{2}-d^{2}\right)}{\left(\xi_{0}-d\right)^{2}+\eta_{0}^{2}} x+\frac{\frac{1}{4}\left(r^{2}-d^{2}\right)^{2}-\eta_{0}^{2} r^{2}}{\left(\xi_{0}-d\right)^{2}+\eta_{0}^{2}}=0 .
$$

Before writing down the solutions of (9) we are introducing the expression

$$
R\left(\xi_{0}, \eta_{0}\right)=4 r^{2}\left(\left(\xi_{0}-d\right)^{2}+\eta_{0}^{2}\right)-\left(r^{2}-d^{2}\right)^{2} .
$$

\section{Lemma 1}

$R\left(\xi_{0}, \eta_{0}\right)$ is positive for $\left(\xi_{0}, \eta_{0}\right)$ with $\xi_{0}^{2}+\eta_{0}^{2}=r^{2}$.

Proof: The expression $\left(\xi_{0}-d\right)^{2}+\eta_{0}^{2}$ is bounded below according to (8). Thus

$$
R\left(\xi_{0}, \eta_{0}\right) \geq 4 r^{2}(r-|d|)^{2}-(r-|d|)^{2}(r+|d|)^{2} \geq(r-|d|)^{2}\left(4 r^{2}-(r+|d|)^{2}\right) .
$$

For the expression $4 r^{2}-(r+|d|)^{2}$ holds

$$
4 r^{2}-(r+|d|)^{2}=(2 r-(r+|d|))(2 r+(r+|d|))=(r+|d|)(3 r+|d|)>0 .
$$

Therefore $R\left(\xi_{0}, \eta_{0}\right)$ is positive for $\left(\xi_{0}, \eta_{0}\right)$ with $\xi_{0}^{2}+\eta_{0}^{2}=r^{2}$.

With expression (10) the solutions of (9) have the form

$$
x_{1,2}=\frac{1}{2\left(\left(\xi_{0}-d\right)^{2}+\eta_{0}^{2}\right)}\left(\left(\xi_{0}-d\right)\left(r^{2}-d^{2}\right) \pm \eta_{0} \sqrt{R\left(\xi_{0}, \eta_{0}\right)}\right),
$$


where the index 1 of $x$ shall correspond to the +sign and the index 2 to the -sign. Substituting (11) into (6) we obtain

$$
y_{1,2}=\frac{1}{2\left(\left(\xi_{0}-d\right)^{2}+\eta_{0}^{2}\right)}\left(\eta_{0}\left(r^{2}-d^{2}\right) \mp\left(\xi_{0}-d\right) \sqrt{R\left(\xi_{0}, \eta_{0}\right)}\right),
$$

where the index 1 of $y$ corresponds to the - sign and the index 2 to the + sign. For $i \in\{1,2\}$ this makes for $x_{i}^{2}+y_{i}^{2}=r^{2}$.

Although the derivation of (11) was carried out under the assumption $\eta_{0} \neq 0$, the case $\eta_{0}=0$ can be recovered from (11). For $\eta_{0}=0$ follows $\xi_{0}= \pm r$. For $\xi_{0}=+r$ we get $x_{1}=\frac{1}{2}(r+d)$ and for $\xi_{0}=-r$ we have $x_{2}=-\frac{1}{2}(r-d)$. In each case we are getting from $x_{1,2}^{2}+y_{1,2}^{2}=r^{2}$ two $y$-values:

$y_{1}= \pm \frac{1}{2} \sqrt{(3 r+d)(r-d)}$ and $y_{2}= \pm \frac{1}{2} \sqrt{(3 r-d)(r+d)}$.

From (11) and (12) we can deduce for $i \in\{1,2\}$ :

$$
\left(\xi_{0}-d\right) x_{i}+\eta_{0} y_{i}=\frac{1}{2}\left(r^{2}-d^{2}\right) .
$$

\section{Construction of the Gardner Ellipse}

The term "Gardner ellipse" has been used in [1] for the ellipse constructed by folding disks, going back to Gardner's publication [4]. In order to calculate the point of intersection $\left(x_{B}, y_{B}\right)$ of the straight line through $C=(0,0)$ and $A_{0}=\left(\xi_{0}, \eta_{0}\right)$ and straight line (2) we are looking at the linear system

$$
\begin{aligned}
& \eta_{0} x_{B}-\xi_{0} y_{B}=0 \\
& \left(\xi_{0}-d\right) x_{B}+\eta_{0} y_{B}=\frac{1}{2}\left(r^{2}-d^{2}\right) .
\end{aligned}
$$

For the determinant $D_{B}$ of the linear system (14) holds:

$$
D_{B}=\eta_{0}^{2}+\left(\xi_{0}-d\right) \xi_{0}=\eta_{0}^{2}+\xi_{0}^{2}-\xi_{0} d=r^{2}-\xi_{0} d
$$

and we have the following positive lower bound

$$
D_{B}=r^{2}-\xi_{0} d \geq r^{2}-\left|\xi_{0}\right||d| \geq r^{2}-r|d|=r(r-|d|)>0 .
$$

The solutions of the linear system (14) are

$$
x_{B}=\frac{\xi_{0}\left(r^{2}-d^{2}\right)}{2\left(r^{2}-\xi_{0} d\right)}, y_{B}=\frac{\eta_{0}\left(r^{2}-d^{2}\right)}{2\left(r^{2}-\xi_{0} d\right)} .
$$

Since $B=\left(x_{B}, y_{B}\right)$ is lying on straight line (2), the perpendicular bisector of $\overline{A_{0} D}$, the line segments $\overline{D B}$ and $\overline{A_{0} B}$ have the same length. Thus

$$
\overline{D B}+\overline{B C}=\overline{A_{0} B}+\overline{B C}=\overline{A_{0} C}=r \text {. }
$$

See Figure 2.

Letting point $A_{0}=\left(\xi_{0}, \eta_{0}\right)$ move on circle $x^{2}+y^{2}=r^{2}$ we obtain a sequence of points $B=\left(x_{B}, y_{B}\right)$ which have a constant sum of distances from two fixed points $C$ and $D$. According to (16) this sum of distances is equal to $r$. 


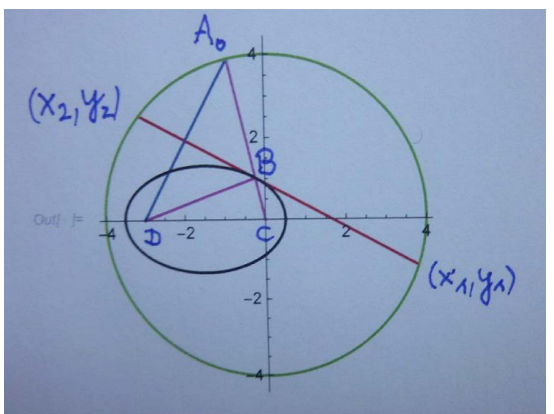

Figure 2. Gardner ellipse with tangent.

Therefore the so generated points are forming an ellipse, the so-called "Gardner ellipse”. It has foci $C$ and $D$ and midpoint $\left(\frac{1}{2} d, 0\right)$. The semi axes of the Gardner ellipse are given by $a=\frac{r}{2}$ and $b=\frac{1}{2} \sqrt{r^{2}-d^{2}}$. The formula of the ellipse is

$$
\frac{\left(x-\frac{d}{2}\right)^{2}}{a^{2}}+\frac{y^{2}}{b^{2}}=1 .
$$

The coordinates of $B=\left(x_{B}, y_{B}\right)$ given in (15) fulfill Equation (17), if the values for the semi axes $a$ and $b$ are substituted.

The tangent to the ellipse (17) at the point $\left(x_{B}, y_{B}\right)$ has the form

$$
\frac{\left(x_{B}-\frac{d}{2}\right)\left(x-\frac{d}{2}\right)}{a^{2}}+\frac{y_{B} y}{b^{2}}=1 .
$$

\section{Proposition 1}

The tangent to ellipse (17) at the point $\left(x_{B}, y_{B}\right)$, given by (18), is equivalent to the perpendicular bisector (2) of line segment $\overline{A_{0} D}$.

Proof: From (15) we get

$$
x_{B}-\frac{d}{2}=\frac{\xi_{0}\left(r^{2}-d^{2}\right)-d\left(r^{2}-\xi_{0} d\right)}{2\left(r^{2}-\xi_{0} d\right)}=\frac{r^{2}\left(\xi_{0}-d\right)}{2\left(r^{2}-\xi_{0} d\right)}
$$

and

$$
\frac{x_{B}-\frac{d}{2}}{a^{2}}=2 \frac{\xi_{0}-d}{r^{2}-\xi_{0} d}, \quad \frac{y_{B}}{b^{2}}=2 \frac{\eta_{0}}{r^{2}-\xi_{0} d} .
$$

This leads to

$$
\begin{gathered}
\frac{\left(x_{B}-\frac{d}{2}\right)\left(x-\frac{d}{2}\right)}{a^{2}}+\frac{y_{B} y}{b^{2}}=2 \frac{\xi_{0}-d}{r^{2}-\xi_{0} d}\left(x-\frac{d}{2}\right)+2 \frac{\eta_{0}}{r^{2}-\xi_{0} d} y=1, \\
\left(\xi_{0}-d\right)\left(x-\frac{d}{2}\right)+\eta_{0} y=\frac{1}{2}\left(r^{2}-\xi_{0} d\right), \\
\left(\xi_{0}-d\right) x+\eta_{0} y=\frac{1}{2}\left(r^{2}-\xi_{0} d\right)+\frac{d}{2}\left(\xi_{0}-d\right)=\frac{1}{2}\left(r^{2}-d^{2}\right),
\end{gathered}
$$


which is the perpendicular bisector (2) of line segment $\overline{A_{0} D}$.

Remark: Because of the relations for the semi axes of the Gardner ellipse $a=\frac{r}{2}$ and $b=\frac{1}{2} \sqrt{r^{2}-d^{2}}$ an arbitrary ellipse with semi axes $a$ and $b$ can be interpreted as Gardner ellipse, choosing $r=2 a$ as radius of the surrounding circle and $d=\sqrt{r^{2}-(2 b)^{2}}=2 \sqrt{a^{2}-b^{2}}$ as distance of the foci.

\section{Bidirectional Folding}

Having obtained the points $\left(x_{1}, y_{1}\right)$ and $\left(x_{2}, y_{2}\right)$ according to (11) and (12) the second fold is starting from $\left(x_{1}, y_{1}\right)$ and the third fold from $\left(x_{2}, y_{2}\right)$, described by Equations (3) and (4) respectively.

The calculation of the points $A_{1}=\left(\xi_{1}, \eta_{1}\right)$ and $A_{2}=\left(\xi_{2}, \eta_{2}\right)$ is combined by looking for $A_{i}=\left(\xi_{i}, \eta_{i}\right)$ for $i \in\{1,2\}$ with

$$
\left(\xi_{i}-d\right) x_{i}+\eta_{i} y_{i}=\frac{1}{2}\left(r^{2}-d^{2}\right), \xi_{i}^{2}+\eta_{i}^{2}=r^{2} .
$$

For $y_{i}=0$, because of $x_{i}= \pm r$, we obtain from (19) for $i \in\{1,2\}$

$$
\xi_{i}=d \pm \frac{1}{2 r}\left(r^{2}-d^{2}\right) \text { and } \eta_{i}= \pm \sqrt{r^{2}-\xi_{i}^{2}} \text {. }
$$

In the case $y_{i} \neq 0$ we use the expansion

$$
\xi_{i}^{2}=\left(\xi_{i}-d+d\right)^{2}=\left(\xi_{i}-d\right)^{2}+2 d\left(\xi_{i}-d\right)+d^{2}
$$

and multiply the second Equation of (19) with $y_{i}^{2}$

$$
\left(\left(\xi_{i}-d\right)^{2}+2 d\left(\xi_{i}-d\right)+d^{2}\right) y_{i}^{2}+\eta_{i}^{2} y_{i}^{2}=r^{2} y_{i}^{2} .
$$

Solving the first Equation of (19) for $\eta_{i} y_{i}$ and substituting in (20) gives

$$
\begin{aligned}
\left(\xi_{i}-d\right)^{2} y_{i}^{2}+ & 2 d y_{i}^{2}\left(\xi_{i}-d\right)+\left(-\left(\xi_{i}-d\right) x_{i}+\frac{1}{2}\left(r^{2}-d^{2}\right)\right)^{2}-\left(r^{2}-d^{2}\right) y_{i}^{2}=0, \\
& \left(\xi_{i}-d\right)^{2}\left(x_{i}^{2}+y_{i}^{2}\right)+\left(2 d y_{i}^{2}-x_{i}\left(r^{2}-d^{2}\right)\right)\left(\xi_{i}-d\right) \\
& +\frac{1}{4}\left(r^{2}-d^{2}\right)^{2}-\left(r^{2}-d^{2}\right) y_{i}^{2}=0 .
\end{aligned}
$$

Because of $x_{i}^{2}+y_{i}^{2}=r^{2}$ we obtain a quadratic equation for $\xi_{i}-d$ :

$$
\begin{aligned}
& \left(\xi_{i}-d\right)^{2}-\frac{2}{r^{2}}\left(\frac{x_{i}}{2}\left(r^{2}-d^{2}\right)-d y_{i}^{2}\right)\left(\xi_{i}-d\right) \\
& +\frac{1}{r^{2}}\left[\left(\frac{1}{2}\left(r^{2}-d^{2}\right)\right)^{2}-\left(r^{2}-d^{2}\right) y_{i}^{2}\right]=0 .
\end{aligned}
$$

Before writing down the solutions of (21) we are introducing the expression

$$
S\left(x_{i}\right)=r^{4}-\left(d x_{i}+\frac{1}{2}\left(r^{2}-d^{2}\right)\right)^{2} .
$$

\section{Lemma 2}

For $i \in\{1,2\}$ and $x_{i}^{2}+y_{i}^{2}=r^{2}$ the expression $S\left(x_{i}\right)$ is positive. 
Proof: For $i \in\{1,2\}$ the expression $S\left(x_{i}\right)$ can be split up in the factors

$$
\begin{aligned}
S\left(x_{i}\right) & =\left[r^{2}-\left(d x_{i}+\frac{1}{2}\left(r^{2}-d^{2}\right)\right)\right]\left[r^{2}+\left(d x_{i}+\frac{1}{2}\left(r^{2}-d^{2}\right)\right)\right] \\
& =\frac{1}{2}\left(r^{2}-2 d x_{i}+d^{2}\right) \frac{1}{2}\left(2\left(r^{2}+d x_{i}\right)+r^{2}-d^{2}\right) .
\end{aligned}
$$

$S\left(x_{i}\right)$ is positive for $x_{i}^{2}+y_{i}^{2}=r^{2}$ with $i \in\{1,2\}$ since

$$
r^{2}-2 d x_{i}+d^{2} \geq r^{2}-2|d| r+d^{2}=(r-|d|)^{2}>0
$$

and

$$
2\left(r^{2}+d x_{i}\right)+r^{2}-d^{2} \geq 2 r(r-|d|)+r^{2}-d^{2}>0 .
$$

With expression (22) the solutions of (21) are

$$
\left(\xi_{i}-d\right)_{ \pm}=\frac{1}{r^{2}}\left[\frac{x_{i}}{2}\left(r^{2}-d^{2}\right)-d y_{i}^{2} \pm y_{i} \sqrt{S\left(x_{i}\right)}\right] .
$$

From (23) we obtain

$$
\left(\xi_{i}\right)_{ \pm}=\frac{1}{r^{2}}\left[x_{i}\left(d x_{i}+\frac{1}{2}\left(r^{2}-d^{2}\right)\right) \pm y_{i} \sqrt{S\left(x_{i}\right)}\right] .
$$

Substituting (23) into the first Equation of (19) yields

$$
\left(\eta_{i}\right)_{\mp}=\frac{1}{r^{2}}\left[y_{i}\left(d x_{i}+\frac{1}{2}\left(r^{2}-d^{2}\right)\right) \mp x_{i} \sqrt{S\left(x_{i}\right)}\right]
$$

or expressed otherwise: (23) and (25) combine to

$$
\left(\xi_{i}-d\right)_{ \pm} x_{i}+\left(\eta_{i}\right)_{\mp} y_{i}=\frac{1}{2}\left(r^{2}-d^{2}\right) .
$$

In addition $\left(\xi_{i}\right)_{ \pm}^{2}+\left(\eta_{i}\right)_{\mp}^{2}=r^{2}$ can be verified.

\section{Interpretation of Solutions}

For $i \in\{1,2\}$ we are looking at the straight lines passing through

$$
\left(\left(\xi_{i}\right)_{+},\left(\eta_{i}\right)_{-}\right) \text {and }\left(\left(\xi_{i}\right)_{-},\left(\eta_{i}\right)_{+}\right) \text {. }
$$

\section{Proposition 2}

For $i \in\{1,2\}$ the straight line

$$
x_{i} x+y_{i} y=x_{i}\left(\xi_{i}\right)_{+}+y_{i}\left(\eta_{i}\right)_{-}
$$

is passing through points (27).

Proof: It is clear that $\left(\left(\xi_{i}\right)_{+},\left(\eta_{i}\right)_{-}\right)$is fulfilling (28). In order to prove

$$
x_{i}\left(\xi_{i}\right)_{-}+y_{i}\left(\eta_{i}\right)_{+}=x_{i}\left(\xi_{i}\right)_{+}+y_{i}\left(\eta_{i}\right)_{-}
$$

we are going to show

$$
x_{i}\left(\left(\xi_{i}\right)_{-}-\left(\xi_{i}\right)_{+}\right)+y_{i}\left(\left(\eta_{i}\right)_{+}-\left(\eta_{i}\right)_{-}\right)=0 .
$$

According to (24) and (25) we have: 


$$
\begin{aligned}
\left(\xi_{i}\right)_{-}-\left(\xi_{i}\right)_{+}= & \frac{1}{r^{2}}\left[x_{i}\left(d x_{i}+\frac{1}{2}\left(r^{2}-d^{2}\right)\right)-y_{i} \sqrt{S\left(x_{i}\right)}\right] \\
& -\frac{1}{r^{2}}\left[x_{i}\left(d x_{i}+\frac{1}{2}\left(r^{2}-d^{2}\right)\right)+y_{i} \sqrt{S\left(x_{i}\right)}\right] \\
= & -\frac{2}{r^{2}} y_{i} \sqrt{S\left(x_{i}\right)}, \\
\left(\eta_{i}\right)_{+}-\left(\eta_{i}\right)_{-}= & \frac{1}{r^{2}}\left[y_{i}\left(d x_{i}+\frac{1}{2}\left(r^{2}-d^{2}\right)\right)+x_{i} \sqrt{S\left(x_{i}\right)}\right] \\
& -\frac{1}{r^{2}}\left[y_{i}\left(d x_{i}+\frac{1}{2}\left(r^{2}-d^{2}\right)\right)-x_{i} \sqrt{S\left(x_{i}\right)}\right] \\
= & \frac{2}{r^{2}} x_{i} \sqrt{S\left(x_{i}\right)} .
\end{aligned}
$$

Thus (29) follows from

$$
x_{i}\left(\left(\xi_{i}\right)_{-}-\left(\xi_{i}\right)_{+}\right)+y_{i}\left(\left(\eta_{i}\right)_{+}-\left(\eta_{i}\right)_{-}\right)=\frac{2}{r^{2}}\left(-x_{i} y_{i}+y_{i} x_{i}\right) \sqrt{S\left(x_{i}\right)}=0 .
$$

Next we are looking at the point of intersection $\left(x_{S}, y_{S}\right)$ of the straight lines (28), i.e. the solution of the following linear system

$$
\begin{aligned}
& x_{1} x_{S}+y_{1} y_{S}=x_{1}\left(\xi_{1}\right)_{+}+y_{1}\left(\eta_{1}\right)_{-} \\
& x_{2} x_{S}+y_{2} y_{S}=x_{2}\left(\xi_{2}\right)_{+}+y_{2}\left(\eta_{2}\right)_{-} .
\end{aligned}
$$

See Figure 3.

\section{Proposition 3}

For the the determinant $D_{S}$ of the linear system (30) holds

$$
D_{S}=x_{1} y_{2}-x_{2} y_{1}=\frac{r^{2}-d^{2}}{2\left(\left(\xi_{0}-d\right)^{2}+\eta_{0}^{2}\right)} \sqrt{R\left(\xi_{0}, \eta_{0}\right)},
$$

which is different from zero for $\left(\xi_{0}, \eta_{0}\right)$ with $\xi_{0}^{2}+\eta_{0}^{2}=r^{2}$.

Proof: According to (11) and (12) we have

$$
\begin{aligned}
& x_{1} y_{2}=\frac{1}{4\left(\left(\xi_{0}-d\right)^{2}+\eta_{0}^{2}\right)}\left(4 r^{2}\left(\xi_{0}-d\right) \eta_{0}+\left(r^{2}-d^{2}\right) \sqrt{R\left(\xi_{0}, \eta_{0}\right)}\right), \\
& x_{2} y_{1}=\frac{1}{4\left(\left(\xi_{0}-d\right)^{2}+\eta_{0}^{2}\right)}\left(4 r^{2}\left(\xi_{0}-d\right) \eta_{0}-\left(r^{2}-d^{2}\right) \sqrt{R\left(\xi_{0}, \eta_{0}\right)}\right) .
\end{aligned}
$$

This gives the result (31).

$D_{S}$ is different from zero for $\left(\xi_{0}, \eta_{0}\right)$ with $\xi_{0}^{2}+\eta_{0}^{2}=r^{2}$ because by Lemma 1 $R\left(\xi_{0}, \eta_{0}\right)$ is positive and $r^{2}-d^{2}>0$ holds.

\section{Theorem 1}

For $i \in\{1,2\}$ holds:

$$
\text { Either }\left(\left(\xi_{i}\right)_{+},\left(\eta_{i}\right)_{-}\right)=\left(\xi_{0}, \eta_{0}\right) \text { or }\left(\left(\xi_{i}\right)_{-},\left(\eta_{i}\right)_{+}\right)=\left(\xi_{0}, \eta_{0}\right) \text {. }
$$

Proof: Because of (13) we have for $i \in\{1,2\}$ 


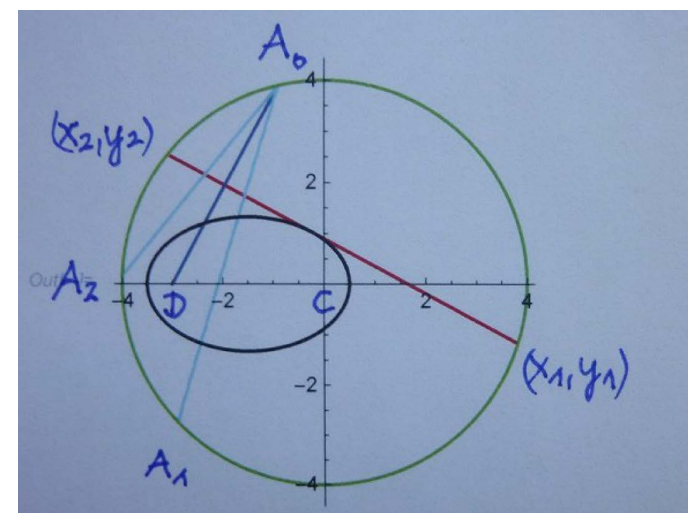

Figure 3. Straight lines through $\left(\left(\xi_{i}\right)_{+},\left(\eta_{i}\right)_{-}\right)$and $\left(\left(\xi_{i}\right)_{-},\left(\eta_{i}\right)_{+}\right)$for $i \in\{1,2\}$.

$$
x_{i} \xi_{0}+y_{i} \eta_{0}=d x_{i}+\frac{1}{2}\left(r^{2}-d^{2}\right) .
$$

Furthermore we have for $i \in\{1,2\}$

$$
x_{i}\left(\xi_{i}\right)_{+}+y_{i}\left(\eta_{i}\right)_{-}=d x_{i}+\frac{1}{2}\left(r^{2}-d^{2}\right)
$$

because (24) and (25) yield

$$
\begin{aligned}
x_{i}\left(\xi_{i}\right)_{+}+y_{i}\left(\eta_{i}\right)_{-} & \frac{x_{i}}{r^{2}}\left[x_{i}\left(d x_{i}+\frac{1}{2}\left(r^{2}-d^{2}\right)\right)+y_{i} \sqrt{S\left(x_{i}\right)}\right] \\
& +\frac{y_{i}}{r^{2}}\left[y_{i}\left(d x_{i}+\frac{1}{2}\left(r^{2}-d^{2}\right)\right)-x_{i} \sqrt{S\left(x_{i}\right)}\right] \\
= & \frac{1}{r^{2}}\left[\left(x_{i}^{2}+y_{i}^{2}\right)\left(d x_{i}+\frac{1}{2}\left(r^{2}-d^{2}\right)\right)+\left(x_{i} y_{i}-y_{i} x_{i}\right) \sqrt{S\left(x_{i}\right)}\right] \\
= & d x_{i}+\frac{1}{2}\left(r^{2}-d^{2}\right) .
\end{aligned}
$$

Combining (33) and (34) we get for $i \in\{1,2\}$

$$
x_{i} \xi_{0}+y_{i} \eta_{0}=x_{i}\left(\xi_{i}\right)_{+}+y_{i}\left(\eta_{i}\right)_{-} \text {. }
$$

This means

$$
\begin{aligned}
& x_{1} \xi_{0}+y_{1} \eta_{0}=x_{1}\left(\xi_{1}\right)_{+}+y_{1}\left(\eta_{1}\right)_{-} \\
& x_{2} \xi_{0}+y_{2} \eta_{0}=x_{2}\left(\xi_{2}\right)_{+}+y_{2}\left(\eta_{2}\right)_{-} .
\end{aligned}
$$

According to Proposition 3 the determinant $D_{S}$ is different from zero for $\left(\xi_{0}, \eta_{0}\right)$ with $\xi_{0}^{2}+\eta_{0}^{2}=r^{2}$. Therefore the linear systems (30) and (35) have one and only one solution, which leads to $\left(x_{S}, y_{S}\right)=\left(\xi_{0}, \eta_{0}\right)$. Point $A_{0}=\left(\xi_{0}, \eta_{0}\right)$ is lying on both straight lines (28) and on the circle $x^{2}+y^{2}=r^{2}$. One of the points of intersection of both straight lines (28) with the circle, $\left(\left(\xi_{i}\right)_{+},\left(\eta_{i}\right)_{-}\right)$ or $\left(\left(\xi_{i}\right)_{-},\left(\eta_{i}\right)_{+}\right)$for $i \in\{1,2\}$, must coincide with $\left(\xi_{0}, \eta_{0}\right)$ what means (32). 


\section{Corollary 1}

With the result of Theorem 1 for the points $A_{i}=\left(\xi_{i}, \eta_{i}\right)$ with $i \in\{1,2\}$ calculated in Section 4 therefore remains the following attribution:

If for $i \in\{1,2\}:\left(\xi_{0}, \eta_{0}\right)=\left(\left(\xi_{i}\right)_{+},\left(\eta_{i}\right)_{-}\right)$, then $\left(\xi_{i}, \eta_{i}\right)=\left(\left(\xi_{i}\right)_{-},\left(\eta_{i}\right)_{+}\right)$.

If for $i \in\{1,2\}:\left(\xi_{0}, \eta_{0}\right)=\left(\left(\xi_{i}\right)_{-},\left(\eta_{i}\right)_{+}\right)$, then $\left(\xi_{i}, \eta_{i}\right)=\left(\left(\xi_{i}\right)_{+},\left(\eta_{i}\right)_{-}\right)$.

With this information and formulas (23) and (25) the following expressions can be evaluated for $i \in\{1,2\}$ :

$$
\begin{gathered}
\left(\xi_{0}-d\right)\left(\xi_{i}-d\right)=\frac{r^{2}-d^{2}}{4 r^{2}}\left(-4 y_{i}^{2}+r^{2}-d^{2}\right) \\
\eta_{0} \eta_{i}=\frac{r^{2}-d^{2}}{4 r^{2}}\left(-4 x_{i}\left(x_{i}-d\right)+r^{2}-d^{2}\right) .
\end{gathered}
$$

Both expressions (36) and (37) combine to

$$
\left(\xi_{0}-d\right)\left(\xi_{i}-d\right)+\eta_{0} \eta_{i}=-\frac{r^{2}-d^{2}}{2 r^{2}}\left(\left(x_{i}-d\right)^{2}+y_{i}^{2}\right) .
$$

\section{The Third Vertex}

We are introducing for $i \in\{1,2\}$ the straight lines

$$
\left(x_{i}-d\right) x+y_{i} y=-\frac{1}{2}\left(\left(x_{i}-d\right)^{2}+y_{i}^{2}\right),
$$

which will have a correspondence with the perpendicular bisectors in (5).

Proposition 4

$$
\begin{aligned}
& \left(x_{2}, y_{2}\right) \text { fulfills }\left(x_{1}-d\right) x+y_{1} y=-\frac{1}{2}\left(\left(x_{1}-d\right)^{2}+y_{1}^{2}\right) . \\
& \left(x_{1}, y_{1}\right) \text { fulfills }\left(x_{2}-d\right) x+y_{2} y=-\frac{1}{2}\left(\left(x_{2}-d\right)^{2}+y_{2}^{2}\right) .
\end{aligned}
$$

Proof: We have to show

$$
\begin{aligned}
& \left(x_{1}-d\right) x_{2}+y_{1} y_{2}=-\frac{1}{2}\left(\left(x_{1}-d\right)^{2}+y_{1}^{2}\right) \\
& \left(x_{2}-d\right) x_{1}+y_{2} y_{1}=-\frac{1}{2}\left(\left(x_{2}-d\right)^{2}+y_{2}^{2}\right) .
\end{aligned}
$$

Transforming the right hand sides of (40) according to $\left(x_{i}-d\right)^{2}+y_{i}^{2}=r^{2}-2 d x_{i}+d^{2}$ with $i \in\{1,2\}$ both statements of (40) can be equivalently transformed to

$$
x_{1} x_{2}+y_{1} y_{2}-d\left(x_{1}+x_{2}\right)=-\frac{1}{2}\left(r^{2}+d^{2}\right) .
$$

It suffices to prove (41). From (11) and (12) we obtain

$$
\begin{gathered}
x_{1} x_{2}=\frac{1}{4\left(\left(\xi_{0}-d\right)^{2}+\eta_{0}^{2}\right)}\left(\left(r^{2}-d^{2}\right)^{2}-4 r^{2} \eta_{0}^{2}\right) \\
y_{1} y_{2}=\frac{1}{4\left(\left(\xi_{0}-d\right)^{2}+\eta_{0}^{2}\right)}\left(\left(r^{2}-d^{2}\right)^{2}-4 r^{2}\left(\xi_{0}-d\right)^{2}\right)
\end{gathered}
$$

leading to 


$$
\begin{aligned}
x_{1} x_{2}+y_{1} y_{2} & =\frac{1}{4\left(\left(\xi_{0}-d\right)^{2}+\eta_{0}^{2}\right)}\left[2\left(r^{2}-d^{2}\right)^{2}-4 r^{2}\left(\left(\xi_{0}-d\right)^{2}+\eta_{0}^{2}\right)\right] \\
& =\frac{\left(r^{2}-d^{2}\right)^{2}}{2\left(\left(\xi_{0}-d\right)^{2}+\eta_{0}^{2}\right)}-r^{2} .
\end{aligned}
$$

Together with

$$
d\left(x_{1}+x_{2}\right)=\frac{2 d\left(\xi_{0}-d\right)\left(r^{2}-d^{2}\right)}{2\left(\left(\xi_{0}-d\right)^{2}+\eta_{0}^{2}\right)}
$$

we get

$$
x_{1} x_{2}+y_{1} y_{2}-d\left(x_{1}+x_{2}\right)=\frac{\left(r^{2}-d^{2}\right)^{2}-2 d\left(\xi_{0}-d\right)\left(r^{2}-d^{2}\right)}{2\left(\left(\xi_{0}-d\right)^{2}+\eta_{0}^{2}\right)}-r^{2} .
$$

The numerator of the fraction in statement (42) can be transformed

$$
\begin{aligned}
\left(r^{2}-d^{2}\right)\left(r^{2}-d^{2}-2 d\left(\xi_{0}-d\right)\right) & =\left(r^{2}-d^{2}\right)\left(r^{2}-2 \xi_{0} d+d^{2}\right) \\
& =\left(r^{2}-d^{2}\right)\left(\left(\xi_{0}-d\right)^{2}+\eta_{0}^{2}\right) .
\end{aligned}
$$

Thus we obtain

$$
x_{1} x_{2}+y_{1} y_{2}-d\left(x_{1}+x_{2}\right)=\frac{1}{2}\left(r^{2}-d^{2}\right)-r^{2}=-\frac{1}{2}\left(r^{2}+d^{2}\right),
$$

which is (41).

Next the point of intersection $\left(x_{T}, y_{T}\right)$ of the straight lines (39) shall be calculated, this means solving the following linear system

$$
\begin{aligned}
& \left(x_{1}-d\right) x_{T}+y_{1} y_{T}=-\frac{1}{2}\left(\left(x_{1}-d\right)^{2}+y_{1}^{2}\right) \\
& \left(x_{2}-d\right) x_{T}+y_{2} y_{T}=-\frac{1}{2}\left(\left(x_{2}-d\right)^{2}+y_{2}^{2}\right) .
\end{aligned}
$$

\section{Proposition 5}

For the determinant $D_{T}$ of the linear system (43) holds

$$
D_{T}=\left(x_{1}-d\right) y_{2}-\left(x_{2}-d\right) y_{1}=\frac{1}{2} \sqrt{R\left(\xi_{0}, \eta_{0}\right)},
$$

which is different from zero for $\left(\xi_{0}, \eta_{0}\right)$ with $\xi_{0}^{2}+\eta_{0}^{2}=r^{2}$.

Proof: Because of (31) we have

$$
x_{1} y_{2}-x_{2} y_{1}=\frac{r^{2}-d^{2}}{2\left(\left(\xi_{0}-d\right)^{2}+\eta_{0}^{2}\right)} \sqrt{R\left(\xi_{0}, \eta_{0}\right)}
$$

and because of (12)

$$
-d\left(y_{2}-y_{1}\right)=-\frac{2 d\left(\xi_{0}-d\right)}{2\left(\left(\xi_{0}-d\right)^{2}+\eta_{0}^{2}\right)} \sqrt{R\left(\xi_{0}, \eta_{0}\right)}
$$

This yields for $D_{T}$ 


$$
D_{T}=\frac{r^{2}-d^{2}-2 d\left(\xi_{0}-d\right)}{2\left(\left(\xi_{0}-d\right)^{2}+\eta_{0}^{2}\right)} \sqrt{R\left(\xi_{0}, \eta_{0}\right)}=\frac{r^{2}-2 d \xi_{0}+d^{2}}{2\left(\left(\xi_{0}-d\right)^{2}+\eta_{0}^{2}\right)} \sqrt{R\left(\xi_{0}, \eta_{0}\right)}
$$

and because of $r^{2}-2 d \xi_{0}+d^{2}=\left(\xi_{0}-d\right)^{2}+\eta_{0}^{2}$ formula (44). That $R\left(\xi_{0}, \eta_{0}\right)$ is positive for $\left(\xi_{0}, \eta_{0}\right)$ with $\xi_{0}^{2}+\eta_{0}^{2}=r^{2}$ was shown in Lemma 1 . Therefore also $D_{T}$ is different from zero for $\left(\xi_{0}, \eta_{0}\right)$ with $\xi_{0}^{2}+\eta_{0}^{2}=r^{2}$.

\section{Proposition 6}

The solutions of the linear system (43) are as follows

$$
x_{T}=d-\frac{\left(\xi_{0}-d\right)\left(r^{2}-d^{2}\right)}{\left(\xi_{0}-d\right)^{2}+\eta_{0}^{2}}, \quad y_{T}=-\frac{\eta_{0}\left(r^{2}-d^{2}\right)}{\left(\xi_{0}-d\right)^{2}+\eta_{0}^{2}} .
$$

Proof: Because $D_{T}$ is different from zero for $\left(\xi_{0}, \eta_{0}\right)$ with $\xi_{0}^{2}+\eta_{0}^{2}=r^{2}$ the linear system (43) has one and only one solution. It is sufficient to show for $i \in\{1,2\}$

$$
\left(x_{i}-d\right)\left(x_{T}-d\right)+y_{i} y_{T}=-\frac{1}{2}\left(\left(x_{i}-d\right)^{2}+y_{i}^{2}\right)-d\left(x_{i}-d\right) .
$$

Substitution of $x_{T}-d$ and $y_{T}$ on the left side of (46) yields

$$
\begin{aligned}
\left(x_{i}-d\right)\left(x_{T}-d\right)+y_{i} y_{T} & =-\frac{\left(x_{i}-d\right)\left(\xi_{0}-d\right)\left(r^{2}-d^{2}\right)}{\left(\xi_{0}-d\right)^{2}+\eta_{0}^{2}}-\frac{y_{i} \eta_{0}\left(r^{2}-d^{2}\right)}{\left(\xi_{0}-d\right)^{2}+\eta_{0}^{2}} \\
& =-\frac{r^{2}-d^{2}}{\left(\xi_{0}-d\right)^{2}+\eta_{0}^{2}}\left(\left(x_{i}-d\right)\left(\xi_{0}-d\right)+y_{i} \eta_{0}\right) .
\end{aligned}
$$

Because of (13) we have

$$
\begin{aligned}
\left(x_{i}-d\right)\left(\xi_{0}-d\right)+y_{i} \eta_{0} & =x_{i}\left(\xi_{0}-d\right)+y_{i} \eta_{0}-d\left(\xi_{0}-d\right) \\
& =\frac{1}{2}\left(r^{2}-d^{2}\right)-d \xi_{0}+d^{2} \\
& =\frac{1}{2}\left(r^{2}-2 d \xi_{0}+d^{2}\right) \\
& =\frac{1}{2}\left(\left(\xi_{0}-d\right)^{2}+\eta_{0}^{2}\right)
\end{aligned}
$$

Therefore we get

$$
\begin{aligned}
\left(x_{i}-d\right)\left(x_{T}-d\right)+y_{i} y_{T} & =-\frac{1}{2}\left(r^{2}-d^{2}\right) \\
& =-\frac{1}{2}\left(r^{2}-2 d x_{i}+d^{2}+2 d x_{i}-2 d^{2}\right) \\
& =-\frac{1}{2}\left(\left(x_{i}-d\right)^{2}+y_{i}^{2}\right)-d\left(x_{i}-d\right) .
\end{aligned}
$$

which is (46).

Proposition 7

$\left(x_{T}, y_{T}\right)$ is located on the circle $x^{2}+y^{2}=r^{2}$.

Proof: From (45) we get

$$
\left(x_{T}-d\right)^{2}+y_{T}^{2}=\frac{\left(r^{2}-d^{2}\right)^{2}\left(\left(\xi_{0}-d\right)^{2}+\eta_{0}^{2}\right)}{\left(\left(\xi_{0}-d\right)^{2}+\eta_{0}^{2}\right)^{2}}=\frac{\left(r^{2}-d^{2}\right)^{2}}{\left(\xi_{0}-d\right)^{2}+\eta_{0}^{2}} .
$$


Because of $x_{T}^{2}=\left(x_{T}-d\right)^{2}+2 d x_{T}-d^{2}$ we have

$$
x_{T}^{2}+y_{T}^{2}=\left(x_{T}-d\right)^{2}+y_{T}^{2}+2 d x_{T}-d^{2}=\frac{\left(r^{2}-d^{2}\right)^{2}}{\left(\xi_{0}-d\right)^{2}+\eta_{0}^{2}}+2 d x_{T}-d^{2} .
$$

Substituting $x_{T}$ from (45) yields

$$
\begin{aligned}
x_{T}^{2}+y_{T}^{2} & =\frac{\left(r^{2}-d^{2}\right)^{2}}{\left(\xi_{0}-d\right)^{2}+\eta_{0}^{2}}+2 d\left[d-\frac{\left(\xi_{0}-d\right)\left(r^{2}-d^{2}\right)}{\left(\xi_{0}-d\right)^{2}+\eta_{0}^{2}}\right]-d^{2} \\
& =\frac{\left(r^{2}-d^{2}\right)^{2}-2 d\left(\xi_{0}-d\right)\left(r^{2}-d^{2}\right)}{\left(\xi_{0}-d\right)^{2}+\eta_{0}^{2}}+d^{2} \\
& =\frac{\left(r^{2}-d^{2}\right)\left(r^{2}-d^{2}-2 d\left(\xi_{0}-d\right)\right)}{\left(\xi_{0}-d\right)^{2}+\eta_{0}^{2}}+d^{2} \\
& =\frac{\left(r^{2}-d^{2}\right)\left(r^{2}-2 d \xi_{0}+d^{2}\right)}{\left(\xi_{0}-d\right)^{2}+\eta_{0}^{2}}+d^{2} \\
& =r^{2}-d^{2}+d^{2}=r^{2} .
\end{aligned}
$$

See Figure 4.

Next we want to show that $\left(x_{T}, y_{T}\right)$ is a point on the perpendicular bisectors of $\overline{D A_{i}}$ for $i \in\{1,2\}$ given in (5).

\section{Proposition 8}

For $i \in\{1,2\}$ holds:

$$
\left(\xi_{i}-d\right) x_{T}+\eta_{i} y_{T}=\frac{1}{2}\left(r^{2}-d^{2}\right) .
$$

Proof: Equation (47) can be equivalently rewritten as

$$
\left(\xi_{i}-d\right)\left(x_{T}-d\right)+\eta_{i} y_{T}=-d\left(\xi_{i}-d\right)+\frac{1}{2}\left(r^{2}-d^{2}\right)=\frac{1}{2}\left(r^{2}-2 d \xi_{i}+d^{2}\right) .
$$

Substituting $x_{T}-d$ and $y_{T}$ according to (45) into the left hand side of (48) we get:

$$
\begin{aligned}
\left(\xi_{i}-d\right)\left(x_{T}-d\right)+\eta_{i} y_{T} & =-\frac{\left(\xi_{i}-d\right)\left(\xi_{0}-d\right)\left(r^{2}-d^{2}\right)}{\left(\xi_{0}-d\right)^{2}+\eta_{0}^{2}}-\frac{\eta_{i} \eta_{0}\left(r^{2}-d^{2}\right)}{\left(\xi_{0}-d\right)^{2}+\eta_{0}^{2}} \\
& =-\frac{r^{2}-d^{2}}{\left(\xi_{0}-d\right)^{2}+\eta_{0}^{2}}\left[\left(\xi_{i}-d\right)\left(\xi_{0}-d\right)+\eta_{i} \eta_{0}\right]
\end{aligned}
$$

The expression in square brackets of (49) is substituted by (38); this yields

$$
=\frac{\left(r^{2}-d^{2}\right)^{2}\left(\left(x_{i}-d\right)^{2}+y_{i}^{2}\right)}{2 r^{2}\left(\left(\xi_{0}-d\right)^{2}+\eta_{0}^{2}\right)}=\frac{\left(r^{2}-d^{2}\right)^{2}\left(r^{2}-2 d x_{i}+d^{2}\right)}{2 r^{2}\left(r^{2}-2 d \xi_{0}+d^{2}\right)} .
$$

Statement (48) is proven if we can show for the expression on the right hand side of (50)

$$
\frac{\left(r^{2}-d^{2}\right)^{2}\left(r^{2}-2 d x_{i}+d^{2}\right)}{2 r^{2}\left(r^{2}-2 d \xi_{0}+d^{2}\right)}=\frac{1}{2}\left(r^{2}-2 d \xi_{i}+d^{2}\right) .
$$




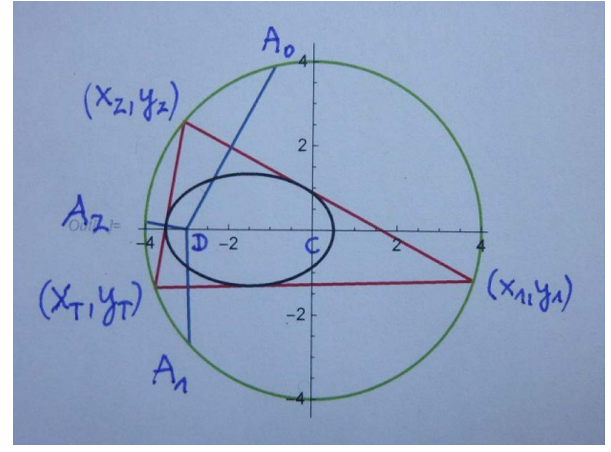

Figure 4. The third vertex $\left(x_{T}, y_{T}\right)$ located on the circle.

This means

$$
\left(r^{2}-d^{2}\right)^{2}\left(r^{2}-2 d x_{i}+d^{2}\right)=r^{2}\left(r^{2}-2 d \xi_{0}+d^{2}\right)\left(r^{2}-2 d \xi_{i}+d^{2}\right) .
$$

Statement (51) will be equivalently transformed. The right hand side of (51) can be expanded

$$
\begin{aligned}
& r^{2}\left(r^{2}-d^{2}-2 d\left(\xi_{0}-d\right)\right)\left(r^{2}-d^{2}-2 d\left(\xi_{i}-d\right)\right) \\
& =r^{2}\left[\left(r^{2}-d^{2}\right)^{2}-2 d\left(r^{2}-d^{2}\right)\left(\xi_{0}-d+\xi_{i}-d\right)+4 d^{2}\left(\xi_{0}-d\right)\left(\xi_{i}-d\right)\right] .
\end{aligned}
$$

Subtracting $r^{2}\left(r^{2}-d^{2}\right)^{2}$ from the left hand side of (51) and from (52) we get

$$
\begin{aligned}
& \left(r^{2}-d^{2}\right)^{2}\left(-2 d x_{i}+d^{2}\right) \\
& =r^{2}\left[-2 d\left(r^{2}-d^{2}\right)\left(\xi_{0}+\xi_{i}-2 d\right)+4 d^{2}\left(\xi_{0}-d\right)\left(\xi_{i}-d\right)\right] .
\end{aligned}
$$

Substituting on the right hand side of (53)

$$
\xi_{0}+\xi_{i}-2 d=\frac{2}{r^{2}}\left(x_{i}\left(d x_{i}+\frac{1}{2}\left(r^{2}-d^{2}\right)\right)-r^{2} d\right)=\frac{2}{r^{2}}\left(\frac{1}{2} x_{i}\left(r^{2}-d^{2}\right)-d y_{i}^{2}\right)
$$

according to Corollary 1 and (24) as well as $\left(\xi_{0}-d\right)\left(\xi_{i}-d\right)$ according to (36) we get

$$
-4 d\left(r^{2}-d^{2}\right)\left(\frac{1}{2} x_{i}\left(r^{2}-d^{2}\right)-d y_{i}^{2}\right)+d^{2}\left(r^{2}-d^{2}\right)\left(-4 y_{i}^{2}+r^{2}-d^{2}\right)
$$

The expression $-4 d^{2}\left(r^{2}-d^{2}\right) y_{i}^{2}$ vanishes in (54). So we have for (53)

$$
\begin{aligned}
\left(r^{2}-d^{2}\right)^{2}\left(-2 d x_{i}+d^{2}\right) & =-4 d\left(r^{2}-d^{2}\right) \frac{1}{2} x_{i}\left(r^{2}-d^{2}\right)+d^{2}\left(r^{2}-d^{2}\right)^{2} \\
& =-2 d\left(r^{2}-d^{2}\right)^{2} x_{i}+d^{2}\left(r^{2}-d^{2}\right)^{2} \\
& =\left(r^{2}-d^{2}\right)^{2}\left(-2 d x_{i}+d^{2}\right) .
\end{aligned}
$$

\section{Theorem 2}

The ellipse created by folding of a disk is enclosed by an arbitrary triangle of tangents, the vertices of which are lying on the circle representing the disk. 
Proof: According to Proposition 8 for $i \in\{1,2\}$ the perpendicular bisectors of $\overline{D A_{i}}$

$$
\left(\xi_{i}-d\right) x+\eta_{i} y=\frac{1}{2}\left(r^{2}-d^{2}\right)
$$

are passing through $\left(x_{T}, y_{T}\right)$. Because of their construction in Section 4 they are also going through $\left(x_{i}, y_{i}\right)$. Thus there is a coincidence with the straight lines (39) in the following way: the straight lines

$$
\begin{aligned}
& \left(\xi_{1}-d\right) x+\eta_{1} y=\frac{1}{2}\left(r^{2}-d^{2}\right) \\
& \left(x_{2}-d\right) x+y_{2} y=-\frac{1}{2}\left(\left(x_{2}-d\right)^{2}+y_{2}^{2}\right),
\end{aligned}
$$

having the same points $\left(x_{1}, y_{1}\right)$ and $\left(x_{T}, y_{T}\right)$, are thus identical; the straight lines

$$
\begin{aligned}
& \left(\xi_{2}-d\right) x+\eta_{2} y=\frac{1}{2}\left(r^{2}-d^{2}\right) \\
& \left(x_{1}-d\right) x+y_{1} y=-\frac{1}{2}\left(\left(x_{1}-d\right)^{2}+y_{1}^{2}\right),
\end{aligned}
$$

having the same points $\left(x_{2}, y_{2}\right)$ and $\left(x_{T}, y_{T}\right)$, are thus also identical.

Therefore the ellipse with foci $C=(0,0)$ and $D=(d, 0)$ and large semi axis $a=\frac{r}{2}$ is enclosed by a triangle of tangents

$$
\begin{aligned}
& \left(\xi_{0}-d\right) x+\eta_{0} y=\frac{1}{2}\left(r^{2}-d^{2}\right) \\
& \left(\xi_{1}-d\right) x+\eta_{1} y=\frac{1}{2}\left(r^{2}-d^{2}\right) \\
& \left(\xi_{2}-d\right) x+\eta_{2} y=\frac{1}{2}\left(r^{2}-d^{2}\right)
\end{aligned}
$$

with vertices $\left(x_{1}, y_{1}\right),\left(x_{2}, y_{2}\right)$ and $\left(x_{T}, y_{T}\right)$ lying on the circle $x^{2}+y^{2}=r^{2}$.

\section{Conclusions}

It was the intention to show by elementary means of analytic geometry the enclosure of the Gardner ellipse, created by folds of a disk, with an arbitrary triangle of tangents, the vertices of which are lying on the surrounding circle representing the disk.

Remark: The Figures in this paper were constructed with Mathematica using the initial data $r=4, d=-3, \quad \xi_{0}=-0.9$ and $\eta_{0}=+\sqrt{r^{2}-\xi_{0}^{2}}$.

\section{Conflicts of Interest}

The author declares no conflicts of interest regarding the publication of this paper.

\section{References}

[1] Gorkin, P. and Shaffer, A. (2021) Making Ellipses by Folding Disks. Mathematics 
Magazine, 94, 53-58. https://doi.org/10.1080/0025570X.2021.1849923

[2] Poncelet, J.V. (1865-1866) Traité des propriétés projectives des figures: ouvrage utile à qui s'occupent des applications de la géometrié descriptive et d'opérations géométriques sur le terrain. Vols. 1-2, 2nd Edition, Gauthier-Villars, Paris.

[3] Daepp, U., Gorkin, P. and Mortini, R. (2002) Ellipses and Finite Blaschke Products. The American Mathematical Monthly, 109, 785-795. https://doi.org/10.1080/00029890.2002.11919914

[4] Gardner, M. (1995) New Mathematical Diversions. Revised Edition, Mathematical Association of America, Washington DC. 